\title{
Approaches to the Preparation of Carborane-Containing Carbosilane Entities
}

Rosario Núñez, Arántzazu González, Clara Viñas, Francesc Teixidor, Reijo Sillanpää and Raikko Kivekäs

\section{Supporting Information:}

\section{Experimental Section}

General Details. Elemental analyses were performed using a Carlo Erba EA1108 microanalyzer. IR spectra were recorded from KBr pellets on a Shimadzu FTIR-8300 spectrophotometer. The Electrospray-Ionization mass spectra (ESI-MS) were recorded on a Bruker Esquire 3000 using a source of ionization and a ions trap analyzer. The ${ }^{1} \mathrm{H}$, ${ }^{1} \mathrm{H}\left\{{ }^{11} \mathrm{~B}\right\}$ NMR (300.13 MHz), ${ }^{11} \mathrm{~B}$ NMR (96.29 MHz), ${ }^{13} \mathrm{C}\left\{{ }^{1} \mathrm{H}\right\}$ NMR $(75.47 \mathrm{MHz})$ and

${ }^{29} \mathrm{Si}$ NMR (59.62 MHz) spectra were recorded on a Bruker ARX 300 spectrometer. All NMR spectra were recorded in $\mathrm{CDCl}_{3}$ solutions at $25^{\circ} \mathrm{C}$. Chemical shift values for ${ }^{11} \mathrm{~B}$ NMR spectra were referenced to external $\mathrm{BF}_{3} \cdot \mathrm{OEt}_{2}$, and those for ${ }^{1} \mathrm{H},{ }^{1} \mathrm{H}\left\{{ }^{11} \mathrm{~B}\right\}$, ${ }^{13} \mathrm{C}\left\{{ }^{1} \mathrm{H}\right\} \mathrm{NMR}$ and ${ }^{29} \mathrm{Si}$ NMR spectra were referenced to $\mathrm{SiMe}_{4}$. Chemical shifts are reported in units of parts per million downfield from reference, and all coupling constants are reported in Hertz.

All reactions were performed under an atmosphere of dinitrogen employing standard Schlenk techniques. THF, $\mathrm{Et}_{2} \mathrm{O}$ and toluene were distilled from sodium benzophenone prior to use. Compound 1-Ph-1,2-closo- $\mathrm{C}_{2} \mathrm{~B}_{10} \mathrm{H}_{11}$ (1) was supplied by Katchem Ltd. (Prague) and used as received. Compound $\mathbf{2}$ was synthesized according to the literature. ${ }^{1} n$-BuLi solution (1.6M in hexanes) was purchased from Lancaster or Aldrich; $\mathrm{HSiMe}_{2} \mathrm{Cl}$ and Karstedt's catalyst from ABCR were used as received.

Preparation of 3: To a solution of 1-Ph-1,2- $\mathrm{C}_{2} \mathrm{~B}_{10} \mathrm{H}_{11}$ (1) (250.0 mg, $\left.1.13 \mathrm{mmol}\right)$ in dry THF $(5 \mathrm{~mL})$ at $0^{\circ} \mathrm{C}$ was added a $1.6 \mathrm{M}$ solution of $n$-BuLi in hexane $(0.8 \mathrm{~mL}, 1.28$ 
mmol) dropwise. The mixture was stirred for 30 minutes at room temperature and after cooled to $0{ }^{\circ} \mathrm{C}, \mathrm{HSiMe}_{2} \mathrm{Cl}(0.16 \mathrm{~mL}, 1.41 \mathrm{mmol})$ was added dropwise with stirring. The suspension was stirred at room temperature overnight. After this time, the mixture was quenched with $10 \mathrm{~mL}$ of a aqueous solution saturated with $\mathrm{NH}_{4} \mathrm{Cl}$, transferred to a separatory funnel and diluted with $30 \mathrm{~mL}$ of $\mathrm{Et}_{2} \mathrm{O}$. The aqueous layer was separated and extracted with additional $\mathrm{Et}_{2} \mathrm{O}$. The organic layers were then dried over anhydrous $\mathrm{MgSO}_{4}$ and concentrated in vacuo to give 3 as a white solid. Yield (308.5 mg, $98 \%$ ). ${ }^{1} \mathrm{H}$ NMR: $\delta$ $0.07\left(\mathrm{~d}, \mathrm{~J}(\mathrm{H}, \mathrm{H})=3.7,6 \mathrm{H}, \mathrm{Si}-\mathrm{CH}_{3}\right), 3.67(\mathrm{sp}, \mathrm{J}(\mathrm{H}, \mathrm{H})=3.7,1 \mathrm{H}, \mathrm{Si}-H), 7.34-7.66(\mathrm{~m}, 5 \mathrm{H}$, $\left.\mathrm{C}_{6} H_{5}\right) .{ }^{1} \mathrm{H}\left\{{ }^{11} \mathrm{~B}\right\} \mathrm{NMR}: \delta 0.07\left(\mathrm{~d}, \mathrm{~J}(\mathrm{H}, \mathrm{H})=3.7,6 \mathrm{H}, \mathrm{Si}-\mathrm{CH}_{3}\right), 2.22$ (br s B-H), 2.40 (br s, B-H), 2.87 (br s, B-H), $3.67(\mathrm{sp}, \mathrm{J}(\mathrm{H}, \mathrm{H})=3.7,1 \mathrm{H}, \mathrm{Si}-H), 7.34-7.66\left(\mathrm{~m}, 5 \mathrm{H}, \mathrm{C}_{6} H_{5}\right) .{ }^{29} \mathrm{Si}$ NMR: $\delta$-4.69 (d, $\left.{ }^{1} J(\mathrm{Si}, \mathrm{H})=217.2\right) .{ }^{11} \mathrm{~B}$ NMR: $\delta 2.1\left(\mathrm{~d},{ }^{1} J(\mathrm{~B}, \mathrm{H})=147,1 \mathrm{~B}, \mathrm{~B}(9)\right),-1.8(\mathrm{~d}$, $\left.{ }^{1} J(\mathrm{~B}, \mathrm{H})=148,1 \mathrm{~B}, \mathrm{~B}(12)\right),-7.1\left(\mathrm{~d},{ }^{1} J(\mathrm{~B}, \mathrm{H})=159,4 \mathrm{~B}, \mathrm{~B}(4,5,8,10)\right),-9.4\left(\mathrm{~d},{ }^{1} J(\mathrm{~B}, \mathrm{H})=\right.$ 189, 2B, B(7,11)), -10.6 (d, $\left.{ }^{1} J(\mathrm{~B}, \mathrm{H})=144,2 \mathrm{~B}, \mathrm{~B}(3,6)\right) .{ }^{13} \mathrm{C}\left\{{ }^{1} \mathrm{H}\right\}$ NMR: $\delta$-3.7 $\left(\mathrm{Si}-\mathrm{CH}_{3}\right)$, $73.0\left(C_{\text {cluster }}\right), 82.9\left(C_{\text {cluster }}\right), 128.5\left(\mathrm{Ph}-C_{\text {para }}\right), 130.4\left(\mathrm{Ph}-C_{\text {orto }}\right), 130.9\left(\mathrm{Ph}-C_{\text {meta }}\right), 132.1(\mathrm{Ph}-$ $\left.C_{\text {ipso }}\right)$. FTIR $(\mathrm{NaCl})_{2} \mathrm{~cm}^{-1}: 2572[v(\mathrm{~B}-\mathrm{H})], 2121[v(\mathrm{Si}-\mathrm{H})], 1260\left[\delta\left(\mathrm{Si}_{-}-\mathrm{CH}_{3}\right)\right]$. Anal. Calcd for $\mathrm{C}_{10} \mathrm{H}_{22} \mathrm{~B}_{10} \mathrm{Si}: \mathrm{C}, 43.13 ; \mathrm{H}, 7.96 \%$. Found: C, 43.10; H, 7.88 .

Preparation of 4. Method A: To a solution of 1-Ph-1,2- $\mathrm{C}_{2} \mathrm{~B}_{10} \mathrm{H}_{11}$ (1) (774.7 mg, $3.52 \mathrm{mmol})$ in dry $\mathrm{Et}_{2} \mathrm{O}(5 \mathrm{~mL})$ at $0^{\circ} \mathrm{C}$ was added a $1.6 \mathrm{M}$ solution of $\mathrm{n}-\mathrm{BuLi}$ in hexane (2.2 $\mathrm{mL}, 3.52 \mathrm{mmol})$ dropwise. The mixture was stirred for 40 minutes at room temperature. After cooled to $0{ }^{\circ} \mathrm{C}$, a solution of freshly prepared $2(452.7 \mathrm{mg}, 0.88 \mathrm{mmol})$ in toluene $(7 \mathrm{~mL})$ was added dropwise with stirring. The suspension was stirred for five minutes at $0^{\circ} \mathrm{C}$ and filtered off through Celite. The solvent were removed and the residue treated with cold $\mathrm{Et}_{2} \mathrm{O}(6 \mathrm{~mL})$ to precipitate 4 as a white solid. Yield $(682.1 \mathrm{mg}, 62 \%)$. Method B: Compound 3 (300.0 mg, $1.08 \mathrm{mmol}$ ) was introduced into a Schlenck and heated to $50{ }^{\circ} \mathrm{C}$, subsequently tetravinylsilane $(0.090 \mathrm{~mL}, 0.51 \mathrm{mmol})$ and 2 drops of 
Karstedt catalyst were added. The mixture was stirred at $50^{\circ} \mathrm{C}$ overnight, and after this time the volatiles were evaporated in the vacuum line. Compound 4 was separated by chromatographic workup in $85 \%$ yield.

Hexane vapors diffusion into a $\mathrm{CHCl}_{3}$ solution of $\mathbf{4}$ gave monocrystals suitable for $\mathrm{X}$-ray analysis. ${ }^{1} \mathrm{H}$ NMR: $\delta$-0.13 (s, 24H, Si-CH $H_{3}, 0.14$ (m, 16H, Si-CH2), 7.31-7.64 (m, 20H, $\left.\mathrm{C}_{6} H_{5}\right) .{ }^{1} \mathrm{H}\left\{{ }^{11} \mathrm{~B}\right\}$ NMR: $\delta-0.13\left(\mathrm{~s}, 24 \mathrm{H}, \mathrm{Si}-\mathrm{CH}_{3}\right), 0.14$ (m, 16H, Si-CH $\left.H_{2}\right), 2.19$ (br s, B- $H$ ), 2.42 (br s, B- $H$ ), 2.61 (br s, B- $H$ ), 2.91 (br s, B-H), 7.31-7.64 (m, 20H, $\left.\mathrm{C}_{6} H_{5}\right) .{ }^{29} \mathrm{Si}\left\{{ }^{1} \mathrm{H}\right\}$ NMR: $\delta 10.36$ (s, Si-C $\left.\mathrm{C}_{\text {cluster }}\right), 10.56$ (s, Si $\left.i_{\text {core }}\right) .{ }^{11} \mathrm{~B}$ NMR: $\delta 1.4$ (br s, 1B, B(9)), -2.9 (d, ${ }^{1} J(\mathrm{~B}$, $\mathrm{H})=144,1 \mathrm{~B}, \mathrm{~B}(12)),-8.1\left(\mathrm{~d},{ }^{1} J(\mathrm{~B}, \mathrm{H})=151,4 \mathrm{~B}, \mathrm{~B}(4,5,8,10)\right),-10.1(\mathrm{br}$ s, 4B, $\mathrm{B}(3,6,7,11)) .{ }^{13} \mathrm{C}\left\{{ }^{1} \mathrm{H}\right\}$ NMR: $\delta-3.3\left(\mathrm{Si}-\mathrm{CH}_{3}\right), 2.2\left(\mathrm{Si}-\mathrm{CH}_{2}\right), 8.1\left(\mathrm{Si}-\mathrm{CH}_{2}\right), 76.0\left(C_{\text {cluster }}\right)$, $83.4\left(C_{\text {cluster }}\right), 128.5$ (Ph- $\left.C_{\text {para }}\right), 130.6\left(\mathrm{Ph}-C_{\text {orto }}\right), 131.2\left(\mathrm{Ph}-C_{\text {meta }}\right), 132.8\left(\mathrm{Ph}-C_{\text {ipso }}\right)$. FTIR $(\mathrm{KBr}), \mathrm{cm}^{-1}: 2568[v(\mathrm{~B}-\mathrm{H})], 1253\left[\delta\left(\mathrm{Si}_{-} \mathrm{CH}_{3}\right)\right]$. Anal. Calcd for $\mathrm{C}_{48} \mathrm{H}_{100} \mathrm{~B}_{40} \mathrm{Si}_{5}: \mathrm{C}, 46.11 ; \mathrm{H}$, 8.06 \%. Found: C, 46.30; H, 8.53. MS-Electrospray (ESI), solution of $\mathrm{CHCl}_{3} / \mathrm{CH}_{3} \mathrm{OH}(1: 3)$ $\mathrm{m} / \mathrm{z}$ : calcd for $\mathrm{C}_{48} \mathrm{H}_{100} \mathrm{~B}_{40} \mathrm{Si}_{5}, 1250.2$; found, $1312\left(\mathrm{M}+2 \mathrm{CH}_{3} \mathrm{OH}\right)^{-}$.

\footnotetext{
${ }^{1}$ Seyferth, D.; Kugita, T.; Rheingold, A. L.; Yap, G. P. A. Organometallics 1995, 14, 5362.
} 